\title{
CT scan Positive Finding Pattern of Head Injury at the University of Gondar Hospital; North West Ethiopia
}

\author{
Zerubabel Tegegne Desita ${ }^{1}$, Wossen Mulugeta $^{2}$ \\ ${ }^{1}$ Assistant Professor of Radiology, University of Gondar, ETHIOPIA \\ ${ }^{2}$ Assistant Professor of Ophthalmology, University of Gondar, ETHIOPIA
}

\begin{abstract}
Background: Head injuries rank high among morbidities due to trauma. Computerized tomography is an important modality in the investigation of these cases. However, there is no literature on the importance of computerized tomography in the diagnosis of head injury in Ethiopia. This study therefore is aimed to document the computerized tomographic features of patients with head injury managed at the University of Gondar Teaching Hospital. Materials and Methods: A cross sectional study involving 96 patients with head injury who had CT scan of the head in the UOG hospital over a 12-month period. Results: Most of the patients were male (74\%). Majority (58\%) were in the age range of 20 to 40 years with a mean age of 31yrs. The most common abnormal findings were skull fracture (52\%) and intracerebral hemorrhage and contusions (51\%). It is followed by subdural hemorrhage (33\%) soft tissue swelling 32\% and epidural hemorrhage $10 \%$. Conclusion: Skull fracture and intra cerebral hemorrhage were the most common abnormal findings. This study has demonstrated the importance of CT scan in the evaluation of head injury by giving visibility of intracranial post traumatic injuries in a high proportion of patients which would be difficult to reach in to diagnosis clinically or using skull radiography alone. This obviously will have a significant role in improving patient management. Taking this in to account expansion of CT scan service for moderate to severe head injury patients is recommended in Ethiopia.
\end{abstract}

Key words: CT scan, CT positive findings, Head injury, Intracranial injury, Skull fracture

12/26/2014 Source of Support: Nil, Conflict of Interest: The authors declare that they have no competing interests How to Cite: Desita ZT and Mulugeta W. 2014. CT scan Positive Finding Pattern of Head Injury at the University of Gondar Hospital; North West Ethiopia Malaysian Journal of Medical and Biological Research, 1, 65-72.

This article is is licensed under a Creative Commons Attribution-NonCommercial 4.0 International License.

Attribution-NonCommercial (CC BY-NC) license lets others remix, tweak, and build upon work non-commercially,

and although the new works must also acknowledge \& be non-commercial.

\section{INTRODUCTION}

Head injury can be defined as any alteration in mental or physical functioning related to a blow to the head. It ranks high among morbidities due to trauma (Adeyekun, 2013). The severity of head injuries is most commonly measured with Glasgow Coma Scale (GCS) score. Traditionally, a score of 13-15 indicates mild injury, of 9-12 moderate injury, and a score of 8 or less severe injury (Shoar and Saadat). Direct and counter coupe injuries can result in an intracerebral focal contusion or hemorrhage as well as an extra cerebral hemorrhage. Brain edema, diffuse axonal injury and skull fractures may also occur alongside other injuries. More 
severe and diffuse axonal injury has been found to correlate with vegetative states and the acute onset of coma following injury (Gupta etal, 2011).

Poly trauma due to road traffic accidents (RTA) is a leading cause of head injury in teenagers and young adults in the developed world (Lewis, 2012). Head injuries cause immediate death in $25 \%$ of acute trauma victims. More than half of the cases of head trauma are caused by RTA, leading to $70 \%$ of all deaths due to brain injury (Gupta etel, 2011; Mohammad etel, 2005). Amongst the severely injured patients, majority survives with severe disability and few continue to be in a vegetative state. Increasing age is associated with poorer outcome in patients with head injury. Assaults, falls, sports, blast injuries are other major risk factors (Peshawar, 2011). Presentations vary from normal to confusion and deep coma. Other clinical features include abnormal papillary reactivity, CN VI \& VII palsies, anosmia, and CSF leak (2, Peshawar, 2011). Patients who have intracranial lesions which require urgent operative intervention need urgent diagnosis and intervention for better outcome (Peshawar, 2011). In studies done in some parts of Africa, results show that head injury ranks high from traumatic causes of morbidity and also like in studies from the developed world these researches show that younger and male population is more affected with head injury (Adeyekun, 2013).

In Ethiopia as to the knowledge of the author no population or hospital based study is done on the pattern of head injury. On studies done in the capital Addis Ababa at TAH to assess cause of hospital admissions; gastrointestinal and neuro-surgical patients constituted $75.8 \%$ of all emergency admissions. Among patients with trauma, isolated head injury was major (59.2\%) cause of death. Neurosurgical emergencies had the highest mortality rate $(36.8 \%)$ (Biluts etel, 2009). Irrespective of the highest rate of occurrence and fatality, there is no proper investigation and management of head injury in the country especially out of the capital due to lack of CT scan and trained personnel. In part of Ethiopia ;Gondar, where the study is done ; trauma constituted about $46 \%$ of surgical patients at University of Gondar Hospital (UOGH), which shows a significant burden to the institution (Osman etel, 2003). Head injury is the second cause of surgical admission in UOGH next to intestinal obstruction and accounts $35 \%$ of all neurosurgical admissions. There is high mortality rate in post traumatic neurosurgical patients (36.6\%) compared to elective patients (4.8\%) (Ghebrat, 1997).

Computed tomography (CT) has become the diagnostic modality of choice for head trauma due to its accuracy, reliability, safety, and wide availability in most part of the world (Adeyekun, 2013; Shoar and Saadat). The changes in microcirculation, impaired auto-regulation, cerebral edema, and axonal injury start as soon as head injury occurs and manifest as clinical, biochemical, and radiological changes. Proper therapeutic management of brain injury is based on correct diagnosis and appreciation of the temporal course of the disease process. CT scan detects and precisely localizes the intracranial hematomas, brain contusions, edema and foreign bodies (Pearce, 2012; Lee, 2005; Trauma etel, 2009). Magnetic resonance imaging (MRI) is considered more sensitive than CT for subtle abnormalities. Because CT does not always explain the posttraumatic neurologic examination, MRI is being performed with increasing frequency. Although MRI at a later stage may be of significant prognostic value, the role of early MRI within 48 hours is minimal (Trauma etel, 2009; James, 2010). Skull radiography is the widely available method of imaging for head injury before the era of CT with limited importance in detecting skull fracture but not helpful in evaluation of intracranial structures (Tasker, 2005).

Not all patients with head injury should have CT scan examination. Patients with moderate and severe head injury with GCS 9-12 and below 9 respectively should have a CT scan examination. Patients with mild head injury should have CT scan based on criteria set by additional clinical findings in addition to GCS of 13-14. This includes clinical signs of skull fracture, amnesia, vomiting, posttraumatic seizure, Age $\geq 60 \mathrm{y}$, neurologic deficit and use of anticoagulant therapy by the patient (Shoar and Saadat; Mohammad etel, 2005). This study was conducted to determine pattern of head injury up on CT scan findings so that the local pattern of head injury can be known and evidence based plan can be implemented in the diagnosis and management of head injury locally and nationally. 


\section{METHODS}

Study area: The study is conducted in the university of Gondar hospital which is located in south north part of Ethiopia $727 \mathrm{~km}$ from Addis Ababa.The hospital is a referral and teaching hospital giving a service to urban and rural population of the surrounding to over 5 million populations. The university is one of the centers of excellence in Ethiopia especially in the health sector under the collage of medical and health sciences. The radiology department is one of the many departments in the collage and gives radiologic medical service besides its academic activities being equipped with high-tech radiologic machines including a 4 slice CT scan machine on which the study is done and has radiologists including the one who conducts this research.

Study design: This descriptive cross-sectional study included 96 patients with head injury who had post traumatic findings on CT of the head in the University of Gondar hospital, from September 2011 to September 2012 after ethical permission is obtained from the hospital administration. All consecutive patients who had post traumatic findings on pre contrast CT scans of the head are included in the study irrespective of age and sex. The CT findings were grouped on basis of the principal findings. A 4 slice bright speed CT scan machine made by GE is used and the results are evaluated and recorded by a radiologist. The data is analyzed with SPSS version 20.

\section{Cranial Computed Tomography Scan Technique and Interpretation of Image}

All patients underwent pre contrast CT scan from skull base to the vertex and sequential axial slices of $5 \mathrm{~mm}$ with $1.25 \mathrm{~mm}$ reconstructed image were obtained. Detailed radiological evaluation was done and findings were recorded.

Pneumocranium was assessed by the presence of intracranial air on the CT scan.

Intra-parenchymal contusions may be hemorrhagic or non-hemorrhagic. Diffuse axonal injury was categorized as parenchyma injury with hemorrhage the former seen as multiple small foci of hyper density. Intracerebral hematomas were homogeneously hyper dense with a density of blood (40-60 $\mathrm{HU})$. Parenchymal contusion and laceration was seen as area of hyper density and hypo density with irregular discontinuous outline. These all are categorized together in this study.

The radiological appearance of a typical epidural hematoma was biconvex, lentiform, crescentic or irregular and was heterogeneous in attenuation, containing areas of hyper dense blood clot and dense serum. The typical appearance of subdural hematoma is hyper dense crescent-shaped extraaxial collection with a convex lateral border and concave medial border overlying the cerebral convexity. It appears hyper, iso and hypo dense in acute sub acute and chronic subtypes.

On CT scan, hyper density of acute hemorrhage was visualized in the sulci overlying the cerebral convexities, within the sylvian fissures, basal cisterns, and inter-hemispheric fissure which is called subarachnoid hemorrhage. Similar hyper density of blood is seen with in the ventricles in ventricular hemorrhage.

Diffuse brain edema is considered when there is compression of the lateral and third ventricles and perimesencephalic cistern with prominent cerebral gyri together with homogenously decreased attenuation with grey-white matter interface. Significant midline shift is considered when shift is more than $5 \mathrm{~mm}$ from midline.

Depressed fractures of skull vault were considered to be present when at least one skull bone showed inward displacement, while other fractures categorized as non depressed when no displacement of bone fragment. Indirect signs of basal skull fractures are not taken as evidence of fracture as both infectious and other inflammatory causes can't be excluded. Significant midline shift is considered when a shift by more than $5 \mathrm{~mm}$ measured from the midline. Multifocal involvement is considered in any two different cranial structures injury; scalp, skull or intracranial structures. 


\section{Ethical clearance}

Ethical clearance was obtained from the UOG hospital administration to use the patients' radiologic and epidemiological data for this specific research without mentioning patients' identity at any of the course of the study. Verbal consent is obtained from patients or their relatives when the patients are not clinically fit to do so.

\section{RESULT}

Most of the patients $74 \%$ were male. Majority $58 \%$ were in the age range of 20 to 40 years and only $3 \%$ were above the age of 50 with a mean age of 31 yrs (tables 1-2).

Skull fracture was found on 52\%, of which $32 \%$ was depressed skull fracture and $8 \%$ had pneumocephalus. Soft tissue swelling was found in $32 \%$ of patient (table3). Among those with soft tissue swelling $93 \%$ had skull fracture while $33 \%$ of patients with no soft tissue swelling had skull fracture (table4).

Epidural hematoma is detected in 10\% while subdural hematoma is diagnosed in $36 \%$ of which acute and sub acute subdural hematoma was 33\% and the rest 3\% was chronic (table3). Only $3 \%$ of patients had both epidural and subdural hematoma simultaneously and also only $11 \%$ and $6 \%$ had subdural and epidural hematoma together with skull fracture respectively making the proportion of epidural hematoma associated with skull fracture $60 \%$ ( table5). Subdural hematoma was maximally present in the temporo-parietal region $(49 \%)$, followed by frontal region $(17 \%)$ while epidural hematoma is common in the frontal region $(50 \%)$ followed by temporo parietal region 30(table6).

More than half $(51 \%)$ had parenchymal hemorrhagic or contusion, and $4 \%$ had diffuse brain edema while the rest $45 \%$ had no detected brain parenchymal injury (table3). One third of (33\%) patients had brain parenchymal focal injury together with skull fracture (table4). Parenchymal hemorrhage was equivalent in both sides $18 ; 36.8 \%$ right and $16 ; 32.6 \%$ left. The right temporoparietal and the left frontal lobes are the commonest site of cerebral hemorrhage and contusion 10; $20.4 \%$ and $8 ; 16.3 \%$ respectively. Bilateral cerebral involvement is seen in 11cases; $22.4 \%$ (table6). Ventricular and subarachnoid hemorrhage was found to be $4 \%$; each $2 \%$. There was focal brain edema in $52.0 \%$ and significant midline shift was found in $62.5 \%$. Overall multifocal involvement was found in $63.5 \%$ (table3).

\section{Discussion}

The neuroradiology of head trauma has undergone dramatic changes since the advent of computed tomography, which has helped significantly to modify the management of head trauma. Using CT scan, this research has confirmed that young males are the most common victim of head injury and a wide variety of $\mathrm{CT}$ abnormalities are identified in head trauma victims at the setup the study conducted. The proportion of intracranial injuries not detectable on skull $x$ ray associated with head trauma is found to be high signifying the high necessity of CT in the setup where the research is done.

The results are consistent with previous studies that have shown that the incidence head trauma was seen in the most productive years of life. A study done in India showed $71 \%$ of the patients belonged to the 20 to 50 years age group (Gupta etal, 2011). In the present study 58\% were in the age range of 20 to 40 years. Males are found to be predominantly affected accounting $74 \%$ similar to in previous studies the male to female ratio was 4:1 (Gupta etal, 2011). Most likely the reason is that males in this age group are more involved to traffic and outdoor activities than females and extreme ages in developing countries and hence most susceptible (Gupta etal, 2011).

Skull fracture was found on $52 \%$, of which $32 \%$ was depressed skull fracture and $4.2 \%$ had pneumocephalus. Almost similar finding is seen in other researches where skull fracture is seen 62\%, and pneumocranium 12\% (Adeyekun, 2013; Gupta etal, 2011). Soft tissue swelling was found in $32 \%$ of patients in this study lower than seen in other study which is up to $86 \%$ being 
the commonest change seen in head injury (Mohammad etel, 2005). This variation can be explained by the difference in the mechanism of injury and the criteria for CT scanning in head injury in different areas based on the economic status and health care service of countries.

In this study epidural hematoma is detected only in $10 \%$ while subdural hematoma in $36 \%$ most being acute and sub acute similar with results on a study done in India where epidural hematoma is $10.9 \%$ and acute subdural hematoma was present in $16.4 \%$ (Mohammad etel, 2005), while subdural hematoma is higher than epidural in other studies (Gupta etal, 2011; Lewis, 2012). In the present study subdural hematoma was maximally present in the temporoparietal region $(49 \%)$, followed by frontal region $(17 \%)$ while epidural hematoma is common in the frontal region (50\%) followed by temporoparietal region $30 \%$. Previous studies stated that epidural hematoma was most common (65\%) in temporo-parietal region (Gupta etal, 2011). In another study done in India the commonest site for subdural and epidural hematoma was found to be in the frontal region (Mohammad etel, 2005). Most epidural hematoma is found associated with skull fracture $(60 \%)$ which is less than seen in other studies: Samudrala et al stated that epidural hematomas are associated with skull fracture in more than $90 \%$ of patients (Gupta etal, 2011).

In the present study, the intracerebral hematoma and cerebral contusion together was found in $51 \%$. As repeated scanning was not possible due to cost and accessibility of CT, cerebral contusion is merged with parenchymal hemorrhage as the former can become evident on follow up scan not seen on the first early scanning hence affecting its frequency. In a study done in India cerebral hemorrhage was found in $46.3 \%$ patients. Cortical contusions were the second most common primary traumatic neuronal injury with initially subtle or normal CT scans in $20 \%$ of the cases, and delayed hemorrhages evident on follow up scan (Gupta etal, 2011).

Parenchymal hemorrhage was equivalent in both sides $18 ; 36.8 \%$ right and $16 ; 32.6 \%$ left. The right temporoparietal and the left frontal lobes are the commonest site of cerebral hemorrhage and contusion 10; $20.4 \%$ and $8 ; 16.3 \%$ respectively. Bilateral cerebral involvement is seen in 11 ; $22.4 \%$. In other studies the frontal and temporoparietal regions are also common sites; $52.5 \%$ (n-93) in the frontal region and 26\% (n-38) in the temporal-parietal region (Gupta etal, 2011; Peshawar, 2011).

The incidence of traumatic subarachnoid hemorrhage, reported by different authors, ranged from $12 \%$ to $44 \%$ (Gupta etal, 2011). In this research subarachnoid and ventricular hemorrhage is found to be less frequent $4 \%$.Midline shift is seen in $31.2 \%$ of the cases in this study while it is 24.5\% in other studies (Gupta etal, 2011).

CT by its ability to accurately differentiate the various forms of gross neuro-pathological lesions, it has led to prompt and non invasive diagnosis. Evidence of brain parenchymal damage on CT is predictive of poor functional outcome. Because of the availability of CT, it was possible to diagnose those intracranial post traumatic changes which are difficult to reach in to conclusion with clinical and skull $x$ ray evidences alone. This improves the management of such patients by large scale. Due to the cost and lack of adequate availability of the CT scan service repeat follow up scans were not frequently done and not included in the study to assess late complications and post treatment outcomes.

\section{Conclusion}

CT scan has shown significant proportion of head injury patients had damage to the brain and other part of head which are hardly visible with skull $x$ ray films. As these lesions are also difficult to differentiate clinically among each other; it would be difficult to intervene surgically for those patients who need it. This shows that the presence of CT scan has made proper management of such patients possible. This research consolidates the essence of expanding CT scan imaging service in Ethiopia as part of improving the health care standard of the country. The mode of 
injury and treatment outcome in relation to the CT scan diagnosis is not studied in this research and is recommended to be studied in another study.

\section{Reference}

Adeyekun AA, Obi-Egbedi-Ejakpovi EB. Computerized topographic patterns in patients with head injury at the university of Benin teaching hospital. Niger J Clin Pract. 2013 Jan-Mar; 16(Adeyekun, 2013):19-22.

Bruce Lee* and Andrew Newberg†. Neuroimaging in Traumatic Brain Imaging NeuroRx. 2005 April; 2(Shoar and Saadat): 372-383. PMCID: PMC1064998

Ghebrat K. Pattern of surgical admissions in Gondar Teaching Hospital, Ethiopia. East Afr Med J. 1997 Dec; 74(12):812-5

Gupta Prashant K1, Krishna Atul2, Dwivedi Amit N1, Gupta Kumkum³, Bala Madhu² , Garg Gouri1 , Agarwal Shivani ${ }^{1}$. CT scan Findings and Outcomes of Head Injury Patients: A Cross Sectional Study. Available in www.jpmsonline.com/issue/Volume 1, Issue 3 (October-December, 2011)

Hagos Biluts, Abebe Bekele, Berhanu Kottiso, Fikre Enqueselassie, Tadios Munie. In-patient surgical mortality in Tikur Anbessa hospital: a five-year review. Ethiopian Medical Journal 2009 Vol. 47 No. 2 pp. $135-142$

J Trauma. Manolakaki D, Velmahos GC, Spaniolas K, de Moya M, Alam HB. Early magnetic resonance imaging is unnecessary in patients with traumatic brain injury. Available from www.ncbi.nlm.nih.gov/pumed. 2009 Apr.

James M. Provenzale. Imaging of Traumatic Brain Injury: A Review of the Recent Medical Literature. AJR, January 2010, Volume 194, Number 1

Khalid Khanzada Peshawar. Pattern of head injury in patients admitted to Neurosurgery Dept. Govt. PGMI/Lady Reading Hospital. KJMS July-December, 2011, Vol. 3, No. 2 -79.

Mark S. Pearce,1 Jane A. Salotti,1 Nicola L. CT Scans in Young People in Great Britain, Radiology Research and Practice. Volume 2012 (2012), Article ID 594278.

Mensur Osman, Yigzaw Kebede, Sissay Anberbir. Magnitude and pattern of injuries in north Gondar administrative zone, northwest Ethiopia. Ethiop Med J. 2003 Jul ;41 (Gupta etal, 2011):213-20 15227886 Cit:2

Mohammad Z, Shameem R, Munawwar H, V.K. Srivastava. A Study of the Pattern of Head Injury in District Aligarh. U.P. India. JIAFM, 2005: 27 (Shoar and Saadat). ISSN 0971-0973103

R C Tasker. Skull x rays, CT scans, and making a decision in head injury. Arch Dis Child 2005; 90:774775 doi:10.1136/adc.2004.067546

Ricki Lewis. CT Scans for Head Injury More Likely for White Children. Available from www.medscap.com, 2012 Aug 08.

Saeed Shoar and Soheil Saadat. Sina Trauma Research Center Tehran University of Medical Sciences Iran .CT Scanning in Minor Head Injury. Available in www.intechopen.com

Table I: Age

\begin{tabular}{|l|r|}
\hline Number & 96 \\
Mean & 31.06 \\
Std. Error of Mean & 1.204 \\
Median & 30.50 \\
Mode & 32 \\
Std. Deviation & 11.795 \\
Variance & 139.112 \\
Skewness & .233 \\
Std. Error of Skewness & .246 \\
Range & 55 \\
Minimum & 7 \\
Maximum & 62 \\
\hline
\end{tabular}


Table II: Sex and Age in Category

\begin{tabular}{|c|c|c|c|}
\hline variable & & frequency & percent \\
\hline sex & male & 71 & 74.0 \\
\cline { 2 - 4 } & female & 25 & 26.0 \\
\hline Age & $1-10$ & 3 & 3.1 \\
& $11-20$ & 14 & 14.6 \\
& $21-30$ & 31 & 32.3 \\
& $31-40$ & 24 & 25.0 \\
& $41-50$ & 21 & 21.9 \\
& $51-60$ & 2 & 2.1 \\
& $>60$ & 1 & 1.0 \\
\hline
\end{tabular}

Table IV: Skull fractures cross tabulation with brain parenchymal and soft tissue injury

\begin{tabular}{|c|c|c|c|c|c|}
\hline $\begin{array}{c}\text { Skull } \\
\text { fracture }\end{array}$ & $\begin{array}{c}\text { Parenchymal } \\
\text { hemorrhage, } \\
\text { laceration and } \\
\text { or contusion }\end{array}$ & $\begin{array}{c}\text { No parenchymal } \\
\text { hemorrhage, } \\
\text { laceration and or } \\
\text { contusion }\end{array}$ & $\begin{array}{c}\text { Diffuse } \\
\text { brain } \\
\text { edema }\end{array}$ & $\begin{array}{c}\text { ST } \\
\text { change } \\
\text { seen }\end{array}$ & $\begin{array}{c}\text { No ST } \\
\text { change }\end{array}$ \\
\hline yes & 32 & 18 & 0 & 29 & 21 \\
\hline no & 17 & 25 & 4 & 2 & 44 \\
\hline Total & 49 & 43 & 4 & 31 & 65 \\
\hline
\end{tabular}

Table III: Types of injury and subtypes (frequency and percentage)

\begin{tabular}{|c|c|c|c|c|c|c|c|}
\hline Type of injury & Ocquirrence & Frequency & Percent & Total & Types & frequency & percent \\
\hline \multirow[t]{4}{*}{ Sktull fracture } & \multirow[t]{3}{*}{ yes } & \multirow[t]{3}{*}{50} & \multirow[t]{3}{*}{52.1} & \multirow[t]{4}{*}{96} & Depressed & 16 & 16.7 \\
\hline & & & & & Non depressed & 30 & 31.3 \\
\hline & & & & & peneumecephalus & 4 & 4.2 \\
\hline & no & 46 & 47.9 & & & & \\
\hline Soft tissue changes & $\begin{array}{l}\text { change seen no } \\
\text { visible ST change }\end{array}$ & $\begin{array}{l}31 \\
65\end{array}$ & $\begin{array}{l}32.3 \\
67.7\end{array}$ & 96 & & & \\
\hline \multirow[t]{5}{*}{ Subdural hem. } & No & 61 & 63.5 & \multirow[t]{5}{*}{96} & & & \\
\hline & \multirow{4}{*}{ yes } & \multirow{4}{*}{35} & \multirow{4}{*}{36.5} & & $\begin{array}{c}\text { Acute/ sub acute } \\
\text { unilateral }\end{array}$ & 23 & 24 \\
\hline & & & & & $\begin{array}{c}\text { Acute/ sub acute } \\
\text { bilateral }\end{array}$ & 9 & 9.4 \\
\hline & & & & & Chronic unilateral & 2 & 2.1 \\
\hline & & & & & Chronic bilateral & 1 & 1 \\
\hline \multirow[t]{3}{*}{ Epidural hem. } & \multirow{2}{*}{ yes } & \multirow[t]{2}{*}{10} & \multirow{2}{*}{10.4} & \multirow[t]{3}{*}{96} & Acute/ sub acute unil. & 7 & 7.3 \\
\hline & & & & & Acute/suba cute bilat & 3 & 3.1 \\
\hline & no & 86 & 89.6 & & & & \\
\hline $\begin{array}{l}\text { Brain } \\
\text { parenchymal } \\
\text { change }\end{array}$ & $\begin{array}{l}\text { - Focal change seen } \\
\text {-No focal or diffuse } \\
\text { edema } \\
\text {-Diffuse brain } \\
\text { edema }\end{array}$ & $\begin{array}{l}49 \\
44 \\
3\end{array}$ & $\begin{array}{c}510 \\
44.8 \\
4.2\end{array}$ & 96 & & & \\
\hline$\widehat{\mathrm{SAH}}$ & $\begin{array}{l}\text { Yes } \\
\text { no }\end{array}$ & $\begin{array}{c}1 \\
95\end{array}$ & $\begin{array}{c}1 \\
99\end{array}$ & 96 & & & \\
\hline vH & \begin{tabular}{|l|} 
Yes \\
no
\end{tabular} & $\begin{array}{c}1 \\
95 \\
\end{array}$ & $\begin{array}{c}1 \\
99 \\
\end{array}$ & 96 & & & \\
\hline multifocal & \begin{tabular}{|l} 
Yes \\
no
\end{tabular} & $\begin{array}{l}61 \\
35\end{array}$ & $\begin{array}{l}63.5 \\
36.5 \\
\end{array}$ & 96 & & & \\
\hline Midline shift & \begin{tabular}{|l} 
Yes \\
no
\end{tabular} & $\begin{array}{l}60 \\
36 \\
\end{array}$ & $\begin{array}{l}62.5 \\
37.5 \\
\end{array}$ & 96 & & & \\
\hline Focal edema & \begin{tabular}{|l} 
Yes \\
no
\end{tabular} & $\begin{array}{l}50 \\
46\end{array}$ & $\begin{array}{l}52.0 \\
48.0\end{array}$ & 96 & & & \\
\hline
\end{tabular}


Table V: Skull fracture cross tabulation with epidural and subdural hematoma

\begin{tabular}{|c|c|c|c|c|c|}
\hline \multicolumn{3}{|c|}{ Skull fracture } & \multicolumn{2}{|c|}{ Subdural hematoma } & \multirow{4}{*}{$\begin{array}{c}\text { Total } \\
44 \\
0 \\
\end{array}$} \\
\hline & & & no & yes & \\
\hline \multirow{3}{*}{ yes } & \multirow{2}{*}{ Epidural hematoma } & no & 33 & 11 & \\
\hline & & yes & 0 & 0 & \\
\hline & Total & & 39 & 11 & 50 \\
\hline \multirow{3}{*}{ no } & \multirow{2}{*}{ Epidural hematoma } & no & 21 & 21 & 42 \\
\hline & & yes & 1 & 3 & 4 \\
\hline & Total & & 22 & 24 & 46 \\
\hline \multirow{3}{*}{ Total } & \multirow{2}{*}{ Epidural hematoma } & no & 54 & 32 & 80 \\
\hline & & yes & 7 & 3 & 10 \\
\hline & Total & & 61 & 35 & 96 \\
\hline
\end{tabular}

Table VI: Site of parenchymal injury, subdural and epidural hematoma (frequency and percentage)

\begin{tabular}{|l|l|c|c|}
\hline Type of injury & Site of injury & Frequency & Percent \\
\hline SDH & Rt frontal & 2 & 2.1 \\
& Rt temporo parietal & 8 & 8.3 \\
& Lt frontal & 4 & 4.2 \\
& Lt temporo parietal & 9 & 9.4 \\
& Bilateral & 12 & 12.5 \\
& Total & 35 & 36.5 \\
\hline EDH & Rt frontal & 4 & 4.2 \\
& Rt temporo parietal & 2 & 2.1 \\
& Lt frontal & 1 & 1.0 \\
& Lt temporo parietal & 1 & 1.0 \\
& Bilateral & 2 & 2.1 \\
& Total & 10 & 10.4 \\
\hline Parenchymal focal injury & Rt frontal & 4 & 4.2 \\
& Rt temporo parietal & 10 & 10.4 \\
& Lt frontal & 8 & 8.3 \\
& Lt temporo parietal & 3 & 3.1 \\
& Rt occipital & 4 & 4.2 \\
& Lt occipital & 5 & 5.2 \\
& Bilateral & 11 & 11.5 \\
& Other site & 4 & 4.2 \\
& Total & 49 & 51.0 \\
\hline
\end{tabular}

Abbreviations: CT(computerized tomography), UOG(University of Gondar), University of Gondar Hospital (UOGH), MRI(Magnetic resonance Imaging), OPD(Out Patient Department), SPSS(), GCS (Glasgow Coma Scale), RTA (road traffic accidents), CN(cranial nerve), CSF(cerebrospinal fluid), TAH(Tikur Anbesa Hospital), GE(General Electronics), HU(Hounce field Unit), $\mathrm{VH}($ Ventricular hemorrhage), SAH(Subarachnoid Hemorrhage), SDH(subdural Hemorrhage), EDH(Epidural hemorrhage)

$$
-0-
$$

